\title{
HCG11 up-regulation induced by ELK4 suppressed proliferation in vestibular schwannoma by targeting miR-620/ELK4
}

\author{
Ruiqing Long ${ }^{1 \dagger}$, Zhuohui Liu ${ }^{1 \dagger}$, Jinghui $\mathrm{Li}^{2}$, Yuan Zhang ${ }^{1}$ and Hualin $\mathrm{Yu}^{2^{*}}$ (D)
}

\begin{abstract}
Background: Vestibular schwannoma (VS) is a kind of benign tumor deriving from the acoustic nerve sheath. Substantial long non-coding RNAs (IncRNAs) were illustrated to have crucial roles in multiple cancers. However, few IncRNAs were elucidated in VS.

Methods: HCG11, miR-620 and ELK4 expression were tested by RT-qPCR. Gain-of-function experiments were conducted to confirm the effect of HCG11 on VS.

Results: HCG11 possessed a low expression in VS cell lines. Overexpression of HCG11 repressed cell proliferation but accelerated apoptosis of VS cells. Moreover, we identified ELK4 stimulated the transcription of HCG11 and their affinity was verified by ChIP assays. MiR-620 was chosen to be a target of HCG11 and it was tested to have a high expression in VS cell lines. Moreover, depletion of miR-620 could inhibit cell proliferative ability while fostering apoptosis rate of VS cells. ELK4 was low expressed in VS cell lines and knockdown of ELK4 could rescue the effects made by HCG11 overexpression on progression of VS.
\end{abstract}

Conclusions: HCG11 could inhibit the growth of VS by targeting miR-620/ELK4 in VS cells. HCG11 was a novel therapeutic target for VS treatment.

Keywords: HCG11, miR-620, ELK4, Vestibular schwannoma

\section{Background}

Acoustic neuroma has another name called vestibular schwannoma (VS). It is a common sighted intracranial tumor but luckily it is benign and has a slow growing pace [1]. But it would become larger and repress the brain and lead to hearing loss, tinnitus and balance disorders as time goes by [2]. VS could threaten life if not treated. The current methods include surgical treatment

\footnotetext{
*Correspondence: hua85815881@163.com

${ }^{\dagger}$ Ruiqing Long and Zhuohui Liu are co-first authors

${ }^{2}$ Neurosurgery Department, The First Affiliated Hospital of Kunming

Medical University, No. 1 Building, No. 295 Xichang Road,

Kunming 650032, Yunnan, China

Full list of author information is available at the end of the article
}

and stereotactic radiotherapy. It will be helpful to cure this disease if the detailed molecular mechanism is understood.

Growing studies indicated that lncRNAs were active regulators in the initiation and development of diverse kinds of cancers $[3,4]$. The aberrant expression of lncRNAs could function as tumor inhibitors or oncogenes [5, 6]. HCG11 was analyzed in hepatocellular carcinoma and was reported as a tumor suppressor [7]. However, the function of HCG11 was not clear in VS.

Mounting essays supported competing endogenous RNAs (ceRNAs) regulatory system could have tremendous effects on the progression of cancers. In this system, lncRNAs acted as sponges of microRNAs (miRNAs) so that their downstream targets could escape from the combination with miRNAs and coded into proteins $[8,9]$.

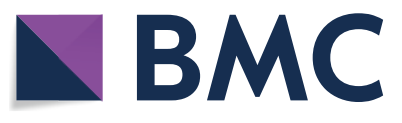

c) The Author(s) 2021. This article is licensed under a Creative Commons Attribution 4.0 International License, which permits use, sharing, adaptation, distribution and reproduction in any medium or format, as long as you give appropriate credit to the original author(s) and the source, provide a link to the Creative Commons licence, and indicate if changes were made. The images or other third party material in this article are included in the article's Creative Commons licence, unless indicated otherwise in a credit line to the material. If material is not included in the article's Creative Commons licence and your intended use is not permitted by statutory regulation or exceeds the permitted use, you will need to obtain permission directly from the copyright holder. To view a copy of this licence, visit http://creativecommons.org/licenses/by/4.0/. The Creative Commons Public Domain Dedication waiver (http://creativecommons.org/publicdomain/zero/1.0/) applies to the data made available in this article, unless otherwise stated in a credit line to the data. 
HCG11 was described to suppress the course of glioma by targeting miR-496/CPEB3 [10]. Nevertheless, regulatory system concerning HCG11 has not been applied and explained in VS till now. In our study, we focused on the role of HCG11 in VS cells.

MiRNAs were introduced to have vital functions in modulating the process of cancers [11-13]. MiR-1 was said to repress cell proliferation in VS by regulating VEGFA [14]. MiR-21 up-regulation was discovered to foster VS proliferation [15]. MiR-620 was analyzed in cervical cancer as an oncogene [16]. However, has the relationship between miR-620 and VS has never been established at all.

ELK4 was reported to exert critical functions in large numbers of articles $[17,18]$. But it has not been studied in VS ever. In this study, we found it as a transcription factor of HCG11 in VS serving as the target of miR-620.

The purpose of our study was to explore the role of HCG11 in VS progression. The rescue assays illustrated the effectiveness of HCG11/miR-620/ELK4 axis in vestibular schwannoma. The axis was displayed as Additional file 1: Figure S1.

\section{Methods}

\section{Tissue collection and cell culture}

Informed consents had acquired from all participants in this clinical study, and protocol had been approved by the Human Studies Committee of the First Affiliated Hospital of Kunming Medical University. The freshly-harvested human tissues of control great auricular nerve (GAN; $\mathrm{n}=50)$ and sporadic VS $(\mathrm{n}=50)$ were collected from indicated surgeries and kept in saline solution, following transporting to laboratory on ice.

\section{Cell culture of normal schwanns cells (SCs) and VSs}

Tissues of control and VS group in sterile PBS were cut to $1 \mathrm{~mm}^{3}$ pieces in DMEM/F12 medium, 1\% penicillin/ streptomycin (Pen/Strep), 10\% fetal bovine serum (FBS) and 1\% GlutaMAX (all, Thermo Fisher Scientific, Inc., Waltham, MA). To acquire purer SC population, epineurium was removed from nerve tissue via tugging and removing the outer layers under dissecting microscope. Tissues were centrifuged at $8{ }^{\circ} \mathrm{C}$ at $3000 \mathrm{~g}$ for $3 \mathrm{~min}$, then the tissue pellets were cultured in new medium adding 5\% Collagenase (Sigma Aldrich, St. Louis, MI) and 0.5\% Hyaluronidase (Sigma-Aldrich) at $37^{\circ} \mathrm{C}$ for 24 h. Human VS cell line HEI-193 was derived from patients with neurofibromatosis type 2 (NF2) and used in this study.

\section{Real-time quantitative PCR (RT-qPCR)}

TRIzol reagent (Thermo Fisher Scientific) was employed for extracting total RNA samples from cells. RNA was converted into cDNA using Reverse Transcription Kit
(Toyobo, Osaka, Japan). SYBR Green Super Mix (BioRad, Hercules, CA) was applied for qPCR. Relative gene expression was standardized to GAPDH or U6, and foldchanges were determined by $2^{-\Delta \Delta C T}$ method.

\section{Cell transfection}

VSs and HEI-193 cells in 24-well plates were reaped for $48 \mathrm{~h}$ of transfection with Lipofectamine 2000 (Invitrogen, Carlsbad, CA). The pcDNA 3.1/HCG11, pcDNA3.1/ ELK4 and relative negative control (NC; termed pcDNA3.1), shRNAs of ELK4 and control (sh-NC), as well as the miR-620 mimics/inhibitor and NC mimics/ inhibitor, all were procured from RiboBio (Guangzhou, China).

\section{Cell proliferation assays}

Cells after transfection were collected and planted to the 96-well plates adding $10 \mu \mathrm{L}$ of CCK-8 (Beyotime, Shanghai, China). Absorbance at $450 \mathrm{~nm}$ was tested by microplate reader to determine cell viability. Besides, the cultured cells were treated with 5-Bromo-20-Deoxyuridine (EdU; Invitrogen) for $20 \mathrm{~h}$ and fixed, in the presence of anti-EdU antibody (AbD Serotec, Munich, Germany).

\section{Cell apoptosis assays}

The transfected VS cells were collected and fixed on ice for $1 \mathrm{~h}$. Then they were stained by Annexin V-APC and 7-AAD, followed by detection with flow cytometry using FACSCalibur (BD Biosciences, San Jose, CA). Besides, terminal deoxynucleotidyl transferase dUTP nick end labeling (TUNEL; Roche, Basel, Switzerland) was applied to assess cell apoptosis for $1 \mathrm{~h}$ at $37^{\circ} \mathrm{C}$ after fixation and permeabilization.

\section{Dual-luciferase reporter assays}

For promoter analysis, HCG11 promoter covering the wild-type (WT) or mutant (Mut) ELK4 binding sites was severally inserted into luciferase reporter vector pGL3 (Promega, Madison, WI), then co-transfected with pcDNA3.1/ELK4 or pcDNA3.1 into VS cells. Besides, the pmirGLO vectors (Promega) containing the WT and Mut interacting sites of miR-620 in HCG11 sequence or ELK4 3'-UTR, were constructed and transfected with indicated plasmids into cells for $48 \mathrm{~h}$. Dual-Luciferase Reporter Assay System (Promega) was used to test luciferase intensity.

\section{UCSC and JASPAR}

UCSC Genome Browser is a website that contains sketched and annotated genomes of various species, including humans, mice and rats, and provides a range of web analytics tools. Thus, we used UCSC to find out the transcription factors of HCG11. In addition, the 
JASPAR database (http://jaspar.genereg.net/) offers open data access collected from publications or experimentally defined results for those who are looking for models for specific transcription factor binding sites. In this study, JASPAR was utilized to predict binding sites of transcription factors on the gene promoters.

\section{ChIP assay}

The crosslinked chromatin was first cut into 200-1000bp fragments for the immunoprecipitation with $2 \mu \mathrm{g}$ of anti-ELK4 or negative control anti-IgG antibody (Millipore, Billerica, MA), along with the $30 \mu \mathrm{L}$ of magnetic beads. Precipitated chromatin DNA after purification was subjected to RT-qPCR.

\section{FISH}

RNA FISH probe for HCG11 was synthesized by Ribobio for incubation with VS cells in hybridization solution. After nuclear counterstaining with DAPI, cells were photographed under fluorescence microscope (Leica, Wetzlar, Germany).

\section{RNA pull-down}

The protein extracts from cultured cells were collected and cultivated with the biotinylated miR-620 (Bio-miR620-Wt/Mut) and Bio-NC. The RNA-protein mixtures collected by beads were analyzed by RT-qPCR.

\section{RIP}

EZ-Magna RIP RNA Binding Protein Immunoprecipitation Kit (Millipore) was utilized for RIP assay in VS cells, in the presence of anti-Argonaute 2 (Ago2) antibody (Millipore) or negative control anti-IgG (Millipore). After isolating the precipitant RNAs, RT-qPCR was followed.

\section{Statistical analyses}

Data were all statistically analyzed by $\mathrm{t}$ test or one-way ANOVA with the help of SPSS version 19.0 (SPSS Inc., Chicago, IL), with the significant level at $\mathrm{p}$-value $<0.05$. Results from 3 or more repeats were displayed as the mean \pm standard deviation (SD).

\section{Results}

\section{HCG11 up-regulation suppressed the proliferation} and fostered apoptosis of VS cells

To have a clear understanding of HCG11 in VS, first we tested the expression of HCG11 in VS tissue. The outcomes of RT-qPCR revealed that the expression of HCG11 was low in VS tissue (Fig. 1a). Then, we detected the expression of HCG11 in VSs and HEl-193 and normal schwanns (SCs) cells. HEl-193 cell was collected from VS patients. Data of RT-qPCR showed that HCG11 harbored a remarkably low expression in VS cell lines compared with normal SCs cells (Fig. 1b). PcDNA3.1/HCG11 was transfected into cells and the results manifested that HCG11 expression was increased by pcDNA3.1/HCG11 (Fig. 1c). The outcome of CCK8 and EdU assays showcased that cell proliferation ability was decreased sharply by HCG overexpression in comparison with negative control (Fig. 1d, e). Apoptosis rate examined by flow cytometry analysis and TUNEL assays were enhanced significantly in comparison with negative control (Fig. 1f, g). In short, HCG11 was low-expressed in VS tissues and cells. And overexpression of HCG11 could inhibit proliferation but promoted apoptosis of VS cells.

\section{ELK4 stimulated transcription of HCG11 in VS cells}

Then, we searched UCSC website (http://genome.uscs. edu.) to find out the transcription factors of HCG11. ELK4 was introduced to have activating transcription function and the motif structure of ELK4 was shown in Fig. 2a from JASPAR (http://jaspar.genereg.net/). The expression of ELK4 was examined by RT-qPCR after adding pcDNA3.1/ELK4. The outcomes disclosed that ELK4 expression was increased (Fig. 2b). To analyze the effect of ELK4 on HCG11, pcDNA3.1/ELK4 was transfected into cells. RT-qPCR data manifested that HCG11 expression was increased distinctly compared with negative control (Fig. 2c). The consequences of luciferase reporter assays displayed that pcDNA3.1/ELK4 could elevate the activity overtly (Fig. 2d). Moreover, results of ChIP experiments exhibited that HCG11 promoters (PMT) were enriched in ELK4 not IgG (Fig. 2e). These results illustrated the interplay between ELK4 and HCG11. To find out the exact position of binding sites, we divided HCG11 PMT binding sites into 6 pieces. The cells received co-transfection with each piece and ELK4. The results of luciferase reporter assays presented that full length of HCG11 PMT, HCG11 PMT 2 (350-800), HCG11 PMT 4 (1150-1600) could enhance the luciferase activity prominently (Fig. 2f). The data from ChIP assays delineated that full length of HCG11, HCG11 PMT 2\&4 had enrichment in ELK4 (Fig. 2g). Therefore, we inferred that there were binding sites in 400-800 domain and 1200-1600 domain. These two parts were marked with 1 and 2. Next, we conducted luciferase reporter assays and the outcomes portrayed that relative luciferase activity was enhanced conspicuously via transfecting pcDNA3.1/ ELK4 and HCG11 PMT WT compared with negative control group. The activities in cells with HCG11 PMT Mut 1 or Mut 2 were weaker than that with HCG11 PMT WT. However, no evident changes could be observed in the plasmid with HCG11 PMT Mut-1/2 (Fig. 2h). In brief, ELK4 could accelerate transcription of HCG11 in VS cells. 


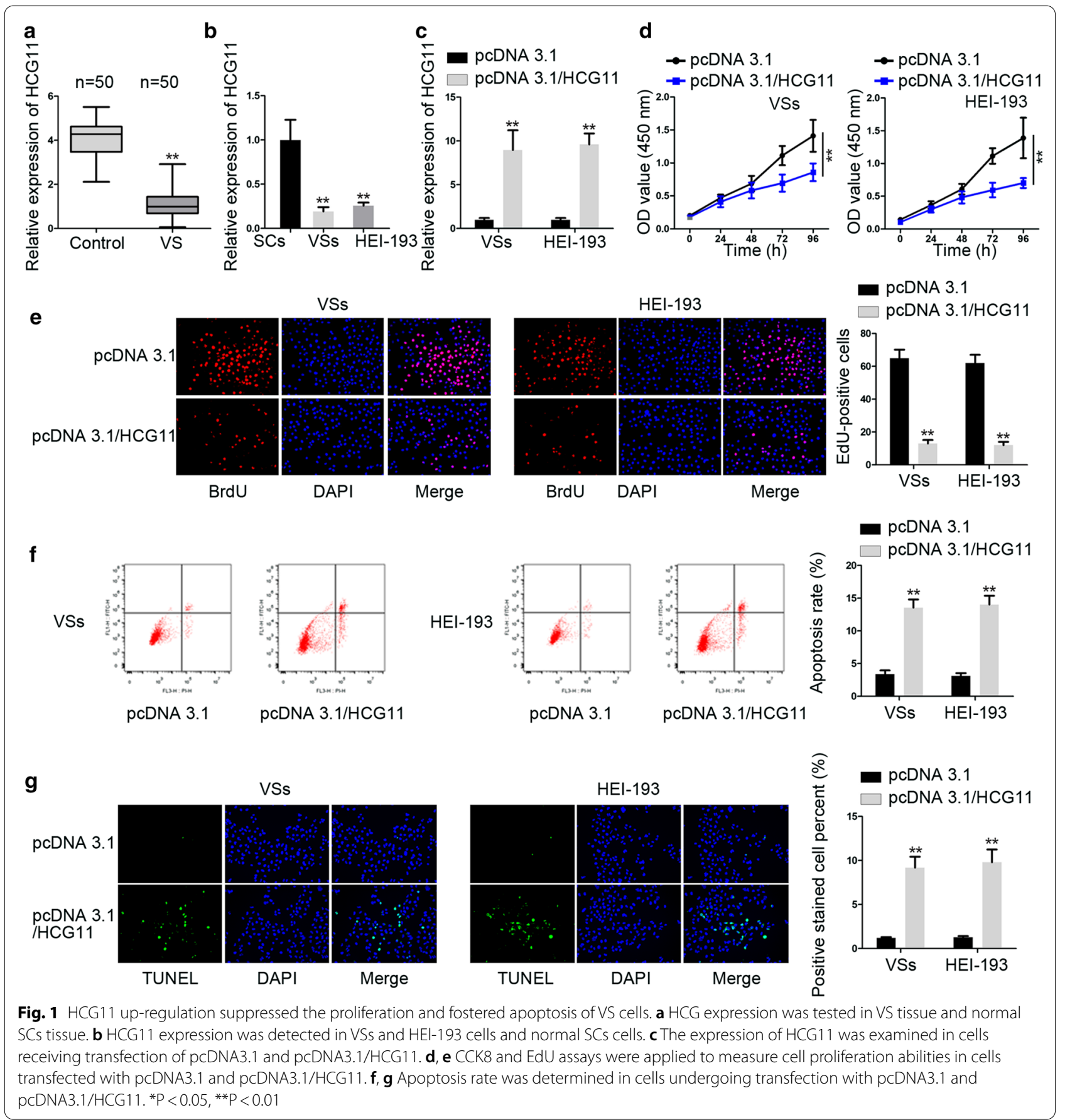

\section{MiR-620 down-regulation suppressed the growth of VS}

Then, pcDNA3.1/HCG11 was transfected into cells to confirm the effect of HCG11 on ELK4 expression. Results from RT-qPCR displayed that ELK4 expression was elevated by pcDNA3.1/HCG11 (Fig. 3a). To explore the role of HCG11 in ceRNA system, first, we applied FISH experiments and the outcomes presented that HCG11 amassed in cytoplasm (Fig. 3b). Then, we used bioinformatics to find that miRNAs had links both with HCG11 and ELK4. As shown in Fig. 3c, there were 14 miRNAs could bind to both HCG11 and ELK4. PCDNA3.1/ELK4 was transfected into cells and results from RT-qPCR showed that only miR-620 expression was declined dramatically by pcDNA3.1/ELK4 (Fig. 3d). The binding sequences were projected by bioinformatics (Fig. 3e). Next, we detected the expression of miR-620 


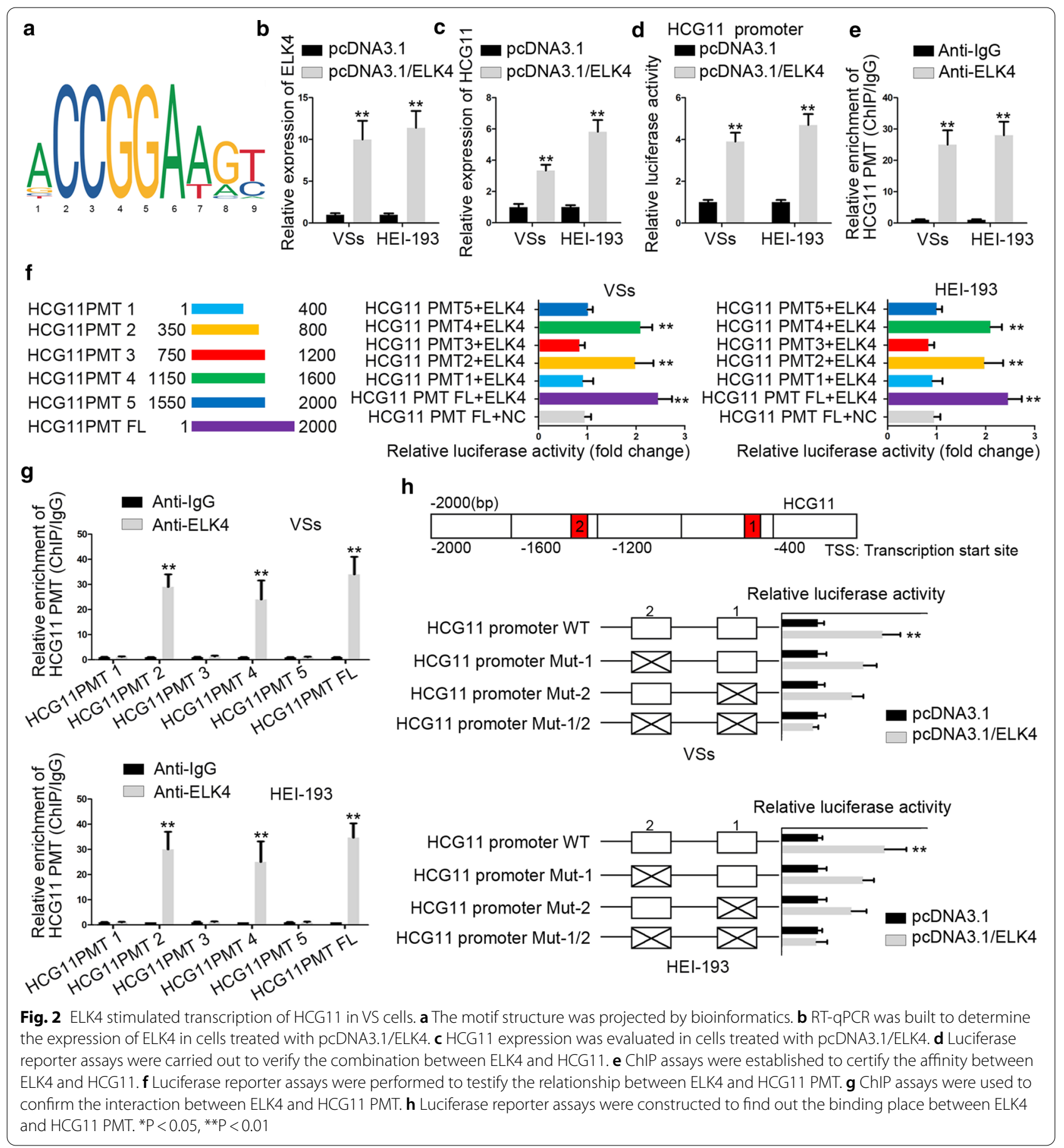

in VS cells and the consequences revealed that miR-620 expression was up-regulated in VS cells (Fig. 3f). The outcomes of RNA pulldown assays indicated that miR-620 could bind to HCG11 (Fig. 3g). MiR-620 mimics were transfected into cells to elevate the expression of miR620 . The data of luciferase reporter assays showed that miR-620 mimics could reduce the activity of plasmid with
HCG11-WT but had no effect on plasmid with HCG11Mut (Fig. 3h). MiR-620 inhibitor was transfected into cells and the expression of miR-620 was diminished by miR-620 inhibitor (Fig. 3i). Then, we studied the effect of miR-620 on VS progression. The consequences of CCK 8 and EdU assays suggested that miR-620 depletion weakened cell proliferation ability (Fig. 3j, k). The apoptosis 
examined by flow cytometry analysis and TUNEL assays was increased by miR-620 down-regulation (Fig. 3l, m). In conclusion, miR-620 could bind to HCG11 and miR620 depletion could inhibit proliferative capacity while enhance apoptosis rate of VS cells.

\section{HCG 11 repressed the process of VS by up-regulating ELK4 expression}

Bioinformatics predicted the binding sites between miR620 and ELK4 (Fig. 4a). RIP assays were set up and the results depicted that HCG11, miR-620 and ELK4 were all abundant in Ago2 antibody but not in IgG antibody (Fig. 4b). Thus, they all coexisted in RNA-induced silencing RNA (RISCs). Data from the luciferase report assays showcased that the luciferase activity of cells with ELK4WT was cut down by miR-620 mimics but there were no distinct changes in that with ELK4-Mut. More interestingly, after HCG11 was transfected into cells, the activity was restored partially (Fig. 4c). Then, the expression of ELK4 was tested in VS cells. The results presented that ELK4 expression was up-regulated in VS cell lines (Fig. 4d). Next, we studied that HCG11 could regulate the process of VS through modulating ELK4. Sh-ELK4\#1 was transfected into cells and data from RT-qPCR described that ELK4 expression was lessened by sh-ELK4\#1 (Fig. 4e). Then, we analyzed whether HCG11 could regulate the process of VS through modulating ELK4. VS cells received transfection with sh-ELK4\#1 and pcDNA3.1/ HCG11. The decreased cell proliferation ability caused by overexpression of HCG11 was rescued by knockdown of ELK4. The results were shown in CCK8 and EdU assays (Fig. 4f, g). The increased apoptosis rate induced by HCG11 up-regulation was restored by silencing ELK4 whose results illuminated in TUNEL and flow cytometry analysis (Fig. 4h, i). In a word, HCG11 constricted cell proliferation while facilitated apoptosis of VS cells via enhancing ELK4 expression.

\section{Discussion}

Vestibular schwannoma (VS) is benign tumors caused by the inactivation of Nf2 gene (27236462). Although the malignant transformation is uncommon (27421984), VS still causes some complications to the patients, such as hearing loss, tinnitus and balance disorder. Currently, the clinical treatment remains limited owing to the lack of effective medicine (27236462). Reviewing previous research, although the diagnosis and treatment regarding eighth carnial nerve schwannoma has achieved improvement over the years, merely few biological factors able to affect tumor growth have been discussed, such as YAP, TAZ and ATEG (28430338). As the prediction of VS growth is likely to determine the therapeutic directions, it is necessary to identify factors able to project tumorigenes to acquire better prognosis.

Recently, lncRNAs are described as targets of treatment in growing studies $[19,20]$, which offered us inspiration to study the role of lncRNAs in regulating VS progression. HCG11 has been studied in breast cancer [21]. In this study, we examined and discovered that HCG11 was in a low expression level in VS cell lines. Overexpression of HCG11 could inhibit proliferative abilities while promote apoptosis of VS cells. This result was similar to the finding in glioma where HCG11 overexpression constricted the proliferation [10].

Then, we analyzed HCG11 in ceRNA network. Accumulating researches suggest that lncRNAs affected the development of cancers via regulating the downstream targets mRNA [22, 23]. HCG11 was reported to work as an anti-oncogene in prostate cancer by sponging miR543 and modulating AKT/mTOR pathway [24]. In our study, first, we used FISH assay to determine the subcellular place of HCG11. Subsequently, we searched bioinformatics and selected out miRNAs which could bind with both HCG11 and ELK4. Finally miR-620 was selected as a target of HCG11. The combination between miR-620 and HCG were certified by RNA pull-down and luciferase report experiments. Moreover, we used luciferase reporter assays to verify the competing relationship between HCG11 and ELK4.

MiRNAs were described to have extremely critical functions in various cancers $[25,26]$. Brazilein was described to induce apoptosis of VS by enhancing miR133a expression [27]. MiR-620 up-regulation was delineated to forward the drug resistance in triple negative breast cancer through regulating DCTD [28]. In this study, miR-620 was examined to be high expressed in

\footnotetext{
(See figure on next page.)

Fig. 3 MiR-620 down-regulation suppressed the growth of VS. a ELK4 expression was examined by RT-qPCR in cells transfected with pcDNA3.1/ HCG11. b FISH experiments were built to judge the place of HCG11 in VS cells. c Venn diagram showed miRNAs could both bind to ELK4 and HCG11. d 14 miRNAs expressions were examined in cells transfected with pcDNA3.1/HCG11. e Bioinformatics projected the binding sequences between HCG11 and ELK4. f MiR-620 expression was appraised in VS cells. $\mathbf{g}$ RNA pull down was constructed to confirm the relationship between HCG11 and miR-620. h RT-qPCR was used to test the expression of miR-620 and luciferase reporter assays were performed to verify the combination between miR-620 and HCG1 1. i RT-qPCR was used to assess the expression of miR-620 in cells treated with miR-620 inhibitor. $\mathbf{j}$, $\mathbf{k}$ CCK8 and EdU assays were built to probe the cell proliferative capacities in cells transfected with pcDNA3.1/HCG11 and miR-620 inhibitor. I, $\mathbf{m}$ The apoptosis rate was estimated in flow cytometry analysis and TUNEL in cells received transfection with pcDNA3.1/HCG11 and miR-620 inhibitor. ${ }^{*} \mathrm{P}<0.05$, ${ }^{* *} \mathrm{P}<0.01$
} 


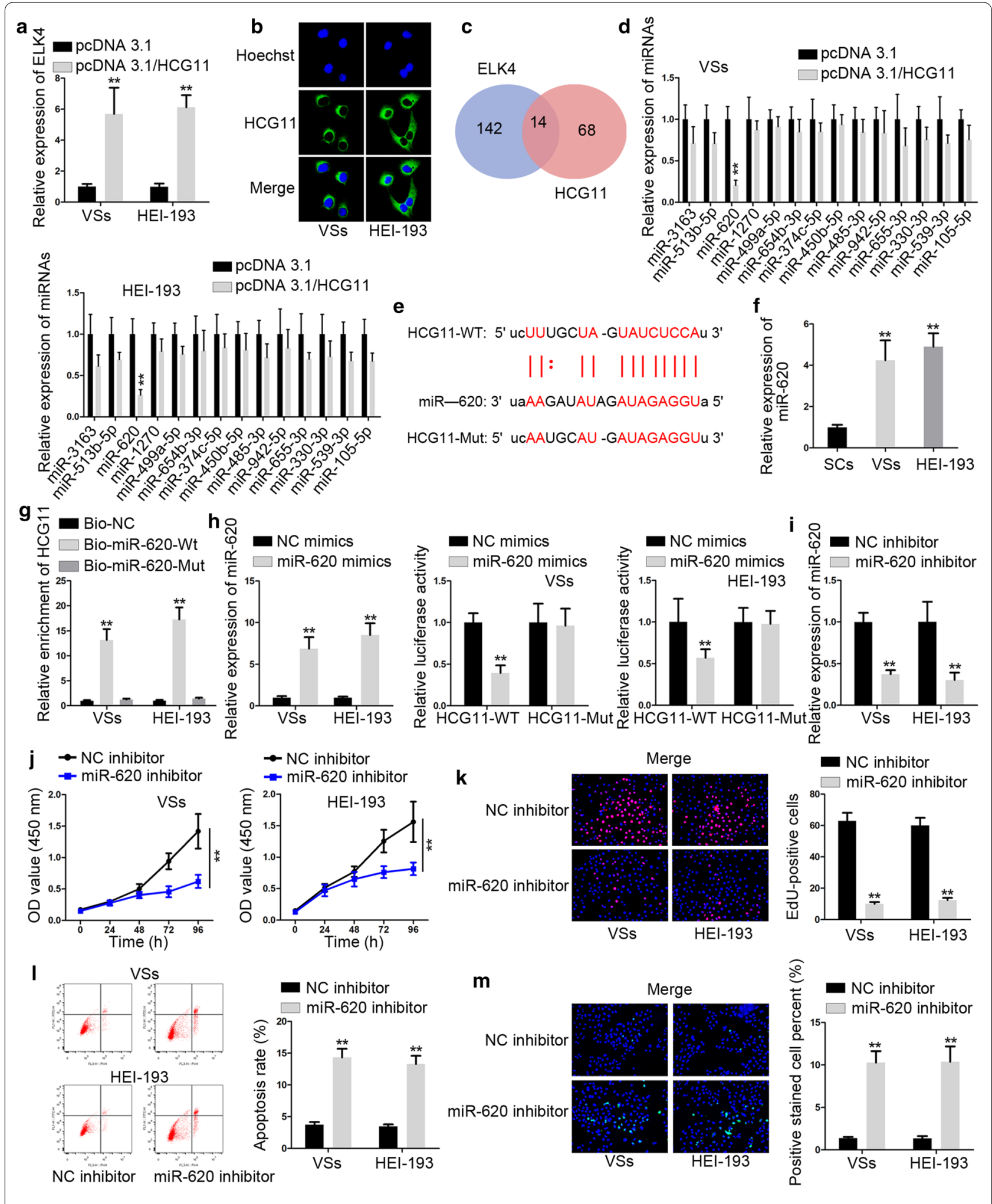




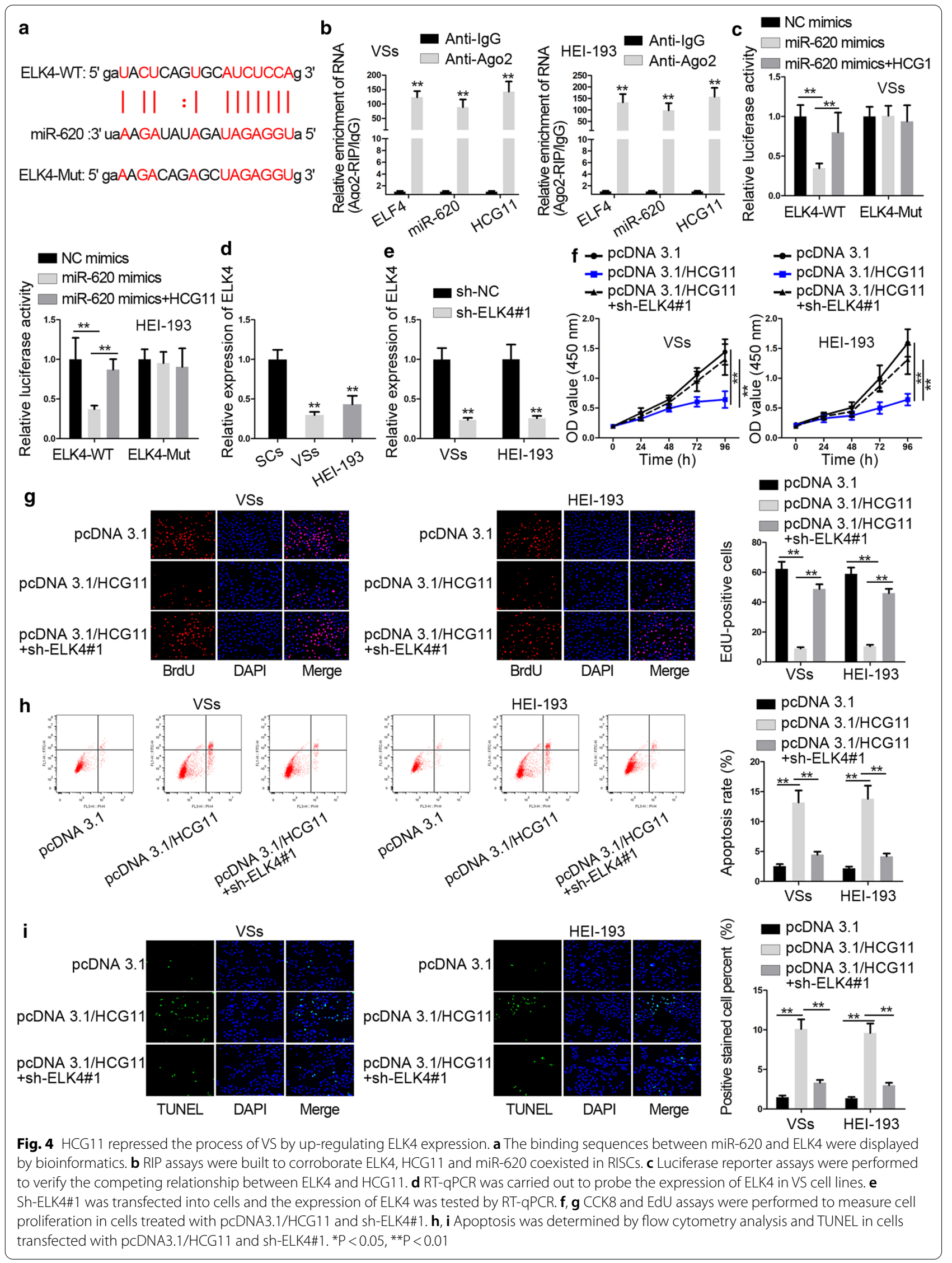


VS cell lines. Depletion of miR-620 could weaken the ability of cell proliferation while accelerated apoptosis rate of VS cell.

ELK4 was reported to have crucial roles in a host of cancers $[29,30]$. In this study, we found ELK4 could regulate the transcription of HCG11 in VS cells. Furthermore, we tested the expression of ELK4 and found it was in a high level in VS cells. In addition, up-regulation of ELK4 could restore the results imposed by HCG11 overexpression in VS progression.

\section{Conclusion}

To sum up, the data collected from our research revealed that HCG11 repressed the course of VS by cooperating miR-620/ELK4. HCG11 could be considered as a target for VS therapy in the future.

\section{Supplementary information}

The online version contains supplementary material available at https://doi. org/10.1186/s12935-020-01691-0.

Additional file 1: Figure S1. The molecular axis of HCG11/miR-620/ELK4 was depicted.

\section{Abbreviations}

VS: Vestibular schwannoma; IncRNAs: Long non-coding RNAs; ceRNAs: Competing endogenous RNAs; miRNAs: MicroRNAs.

\section{Acknowledgements}

We appreciate the supports of all lab workers.

\section{Authors' contributions}

RL contributes to the conception and design of the research; ZL acquired, analyzed and interpreted the experimental data; $J$ and $Y Z$ drafted the work and revised it critically; $\mathrm{HY}$ approved the final version to be published. All authors read and approved the final manuscript.

\section{Funding}

This study was supported by Health Science and Technology Project of Yunnan, China. Number: 2018 NS0156.

\section{Availability of data and materials}

Not applicable.

\section{Ethics approval and consent to participate}

The informed consents had acquired from all participants in this clinical study, and protocol had approved by the Human Studies Committee of the First Affiliated Hospital of Kunming Medical University.

\section{Consent for publication}

Not applicable.

\section{Competing interests}

The authors declare that they have no competing interests.

\section{Author details}

${ }^{1}$ Otolaryngology Department, The First Affiliated Hospital of Kunming Medical University, Kunming 650032, Yunnan, China. ${ }^{2}$ Neurosurgery Department, The First Affiliated Hospital of Kunming Medical University, No. 1 Building, No. 295 Xichang Road, Kunming 650032, Yunnan, China.
Received: 19 May 2020 Accepted: 1 December 2020

Published online: 05 January 2021

\section{References}

1. Jackler RK, Pitts LH. Acoustic neuroma. Neurosurg Clin N Am. 1990;1 (1):199-223.

2. McDonald R. Acoustic neuroma: what the evidence says about evaluation and treatment. J Fam Pract. 2011;60(6):E1-4.

3. Wang J, Su Z, Lu S, Fu W, Liu Z, Jiang X, Tai S. LncRNA HOXA-AS2 and its molecular mechanisms in human cancer. Clin Chim Acta. 2018:485:229-33

4. Thin KZ, Liu X, Feng X, Raveendran S, Tu JC. LncRNA-DANCR: a valuable cancer related long non-coding RNA for human cancers. Pathol Res Pract. 2018:214(6):801-5.

5. Zhang S, Zhong G, He W, Yu H, Huang J, Lin T. IncRNA up-regulated in nonmuscle invasive bladder cancer facilitates tumor growth and acts as a negative prognostic factor of recurrence. J Urol. 2016;196(4):1270-8.

6. Huang HW, Xie H, Ma X, Zhao F, Gao Y. Upregulation of LncRNA PANDAR predicts poor prognosis and promotes cell proliferation in cervical cancer. Eur Rev Med Pharmacol Sci. 2017;21(20):4529-35.

7. Xu Y, Zheng Y, Liu H, LiT. Modulation of IGF2BP1 by long non-coding RNA HCG11 suppresses apoptosis of hepatocellular carcinoma cells via MAPK signaling transduction. Int J Oncol. 2017;51(3):791-800.

8. Shen L, Wang Q, Liu R, Chen Z, Zhang X, Zhou P, Wang Z. LncRNA Inc-RI regulates homologous recombination repair of DNA double-strand breaks by stabilizing RAD51 mRNA as a competitive endogenous RNA. Nucleic Acids Res. 2018;46(2):717-29.

9. Zheng $X$, Liu M, Song Y, Feng C. Long noncoding RNA-ATB impairs the function of tumor suppressor miR-126-mediated signals in endometrial cancer for tumor growth and metastasis. Cancer Biother Radiopharm. 2019:34(1):47-55.

10. Chen Y, Bao C, Zhang X, Lin X, Huang H, Wang Z. Long non-coding RNA HCG11 modulates glioma progression through cooperating with miR496/CPEB3 axis. Cell Prolif. 2019:52:e12615.

11. Mei LL, Qiu YT, Zhang B, Shi ZZ. MicroRNAs in esophageal squamous cell carcinoma: potential biomarkers and therapeutic targets. Cancer Biomarkers. 2017;19(1):1-9.

12. Mao Y, Liu J, Zhang D, Li B. MiR-1290 promotes cancer progression by targeting nuclear factor I/X(NFIX) in esophageal squamous cell carcinoma (ESCC). Biomed Pharmacother. 2015:76:82-93.

13. de Melo Maia B, Rodrigues IS, Akagi EM, Soares do Amaral N, Ling H, Monroig P, Soares FA, Calin GA, Rocha RM. MiR-223-5p works as an oncomiR in vulvar carcinoma by TP63 suppression. Oncotarget. 2016;7(31):49217-31.

14. Li SL, Ma XH, Ji JF, Li H, Liu W, Lu FZ, Wu ST, Zhang Y: miR-1 association with cell proliferation inhibition and apoptosis in vestibular schwannoma by targeting VEGFA. Genet Mol Res. 2016, 15(4).

15. Cioffi JA, Yue WY, Mendolia-Loffredo S, Hansen KR, Wackym PA, Hansen MR. MicroRNA-21 overexpression contributes to vestibular schwannoma cell proliferation and survival. Otol Neurotol. 2010;31(9):1455-62.

16. Tian JDC, Liang L. Involvement of circular RNA SMARCA5/microRNA-620 axis in the regulation of cervical cancer cell proliferation, invasion and migration. European review for medical and pharmacological sciences. 2018;22(24):8589-98

17. Wang H, Zhang H, Zeng J, Tan Y. ceRNA network analysis reveals prognostic markers for glioblastoma. Oncol Lett. 2019;17(6):5545-57.

18. Lee HS, Jung W, Lee E, Chang H, Choi JH, Kim HG, Kim A, Kim BH. SIRT7, H3K18ac, and ELK4 immunohistochemical expression in hepatocellular carcinoma. J Pathol Transl Med. 2016;50(5):337-44.

19. Ding F, Lai J, Gao Y, Wang G, Shang J, Zhang D, Zheng S. NEAT1/miR23a-3p/KLF3: a novel regulatory axis in melanoma cancer progression. Cancer Cell Int. 2019;19:217.

20. Cheng D, Bao C, Zhang $X$, Lin $X$, Huang $H$, Zhao L. LncRNA PRNCR1 interacts with HEY2 to abolish miR-448-mediated growth inhibition in non-small cell lung cancer. Biomed Pharmacother. 2018;107:1540-7.

21. Liu H, Li J, Koirala P, Ding X, Chen B, Wang Y, Wang Z, Wang C, Zhang X, Mo YY. Long non-coding RNAs as prognostic markers in human breast cancer. Oncotarget. 2016;7(15):20584-96. 
22. Liu F, Yuan JH, Huang JF, Yang F, Wang TT, Ma JZ, Zhang L, Zhou CC, Wang F, Yu J, et al. Long noncoding RNA FTX inhibits hepatocellular carcinoma proliferation and metastasis by binding MCM2 and miR-374a. Oncogene. 2016;35(41):5422-34.

23. Yu X, Zhao J, He Y. Long non-coding RNA PVT1 functions as an oncogene in human colon cancer through miR-30d-5p/RUNX2 axis. J BUON. 2018;23(1):48-54.

24. Wang $Y C$, He WY, Dong $C H$, Pei L, Ma YL. IncRNA HCG11 regulates cell progression by targeting miR-543 and regulating AKT/mTOR pathway in prostate cancer. Cell Biol Int. 2019;43(12):1453-62.

25. Ansari MH, Irani S, Edalat H, Amin R, Mohammadi Roushandeh A. Deregulation of miR-93 and miR-143 in human esophageal cancer. Tumour Biol. 2016;37(3):3097-103.

26. Samsonov R, Burdakov V, Shtam T, Radzhabovsmall CZ, Vasilyev D, Tsyrlina E, Titov S, Ivanov M, Berstein L, Filatov M, et al. Plasma exosomal miR-21 and miR-181a differentiates follicular from papillary thyroid cancer. Tumour Biol. 2016;37(9):12011-21.
27. Mou Z, Wang Y, Li Y. Brazilein induces apoptosis and G1/G0 phase cell cycle arrest by up-regulation of miR-133a in human vestibular schwannoma cells. Exp Mol Pathol. 2019;107:95-101.

28. Wu C, Zhao A, Tan T, Wang Y, Shen Z. Overexpression of microRNA-620 facilitates the resistance of triple negative breast cancer cells to gemcitabine treatment by targeting DCTD. Exp Ther Med. 2019;18(1):550-8.

29. Li N, Chen J, Zhao J, Wang T. MicroRNA-3188 targets ETS-domain protein 4 and participates in RhoA/ROCK pathway to regulate the development of atherosclerosis. Pharmazie. 2017;72(11):687-93.

30. Kaikkonen S, Makkonen H, Rytinki M, Palvimo JJ. SUMOylation can regulate the activity of ETS-like transcription factor 4. Biochem Biophys Acta. 2010;1799(8):555-60.

\section{Publisher's Note}

Springer Nature remains neutral with regard to jurisdictional claims in published maps and institutional affiliations.
Ready to submit your research? Choose BMC and benefit from:

- fast, convenient online submission

- thorough peer review by experienced researchers in your field

- rapid publication on acceptance

- support for research data, including large and complex data types

- gold Open Access which fosters wider collaboration and increased citations

- maximum visibility for your research: over 100M website views per year

At $\mathrm{BMC}$, research is always in progress.

Learn more biomedcentral.com/submissions 